\title{
CRIAÇÃO DE PROTOCOLO ELETRÔNICO PARA TERAPIA NUTRICIONAL ENTERAL DOMICILIAR
}

\author{
New electronic protocol for home enteral nutrition therapy \\ Maria Eliana M. SCHIEFERDECKER ${ }^{1}$, Carlos Henrique KURETZKI ${ }^{2}$, Antonio Carlos L. CAMPOS ${ }^{2}$, \\ Osvaldo MALAFAIA ${ }^{2}$, José Simão de Paula PINTO $^{3}$, Nayana Cavassim do NASCIMENTO ${ }^{5}$
}

Trabalho realizado no ${ }^{1}$ Departamento de Nutrição; ${ }^{2}$ Programa de Pós-Graduação em Clínica Cirúrgica; ${ }^{3}$ Programa de PósGraduação em Ciência, Gestão e Tecnologia da Informação e ${ }^{4}$ Curso de Nutrição da Universidade Federal do Paraná, Curitiba, PR, Brasil.

DESCRTORES - Protocolo eletrônico. Nutrição enteral. Serviços de assistência domiciliar.
RESUMO - Racional: A utilização de protocolo com base eletrônica de dados contribui para tornar disponível um grande volume de informações organizadas e estruturadas. A informática pode contribuir no desenvolvimento do conhecimento da Terapia Nutricional Enteral Domiciliar (TNED) por intermédio da pesquisa. Objetivo: Elaborar um protocolo eletrônico para o atendimento de pacientes em TNED. Método: Após a revisão sobre a TNED em material teórico, foram levantados 1793 subitens e agrupados em nove itens principais: Identificação, Avaliação nutricional, Prescrição dietética, Indicações de nutrição enteral, Vias de acesso para nutrição enteral, Composição da nutrição enteral, Sistema de administração, Complicações e Reinternamentos. Definiuse com o nome Protocolo Mestre Informatizado de TNED e foi utilizado o Sistema Integrado de Protocolos Eletrônicos (SINPEC) para sua efetivação. A última etapa foi a criação de um protocolo específico a partir do protocolo mestre. Depois de instalado o programa o sistema reconhece o usuário com a identificação de segurança definida pelo tipo de autorização e disponibiliza a tela para seleção do protocolo mestre que o usuário poderá acessar. Os itens que compõe o protocolo específico estão contidos no Protocolo Mestre e as informações referentes a este protocolos são visualizados. Conclusão: Foi possível criar um protocolo eletrônico para o atendimento de pacientes em TNED com dados seguros que contribuem para a pesquisa nesta área.

\section{Correspondência: \\ Maria Eliana M. Schieferdecker \\ E-mail: meliana@ufpr.br \\ Fonte de financiamento: não há \\ Conflito de interesses: não há}

Recebido para publicação: 01/04/2013 Aceito para publicação: 18/06/20123

HEADINGS - Electronic protocol. Enteral nutrition. Home care services.
ABSTRACT - Background: Informatics can help the development of home enteral nutrition therapy (HENT). By using an electronic protocol with an electronic database it is possible to organize, structure and manage, information. Aim: To develop an electronic protocol for data collection on treatment of patients with home enteral nutrition. Method: After a review in theoretical material about home enteral nutrition therapy, 1793 data collection items were selected and grouped into nine categories: patient identification, nutritional assessment, dietetics prescription, indications for enteral nutrition, access to enteral nutrition options, composition of enteral nutrition, administration system, complications, and readmission. Was used the Electronic Integrated Computerized Procotols System $\left(\mathrm{SINPE}^{\odot}\right)$ to organize that knowledge and the database that attends this research was named Informatized Master Protocol of HENT. The last step was the creation of a specific protocol to collect data by using informations from this database. Results: After installing the program, the system recognizes the user by identifying a pre-defined security authorization and also provides a screen to select a master protocol which the user can access. Items that arrange the specific protocol are contained in the Master Protocol and the information for this protocol are displayed. Conclusion: It was possible to create an electronic protocol for HENT patients care with safety data based on research in enteral nutrition support.

\section{INTRODUÇÃO}

$\mathrm{O}$ desenvolvimento dos computadores digitais teve início nos Estados Unidos e na Europa durante a Segunda Guerra Mundial. Seu uso para fins não-militares começou a partir da metade da década de 50. Nesta época nasceu também a Informática Médica que integra os computadores à Medicina. Com o desenvolvimento do modelo interdisciplinar de tratamento - em que o paciente pode ser atendido por várias especialidades 
diferentes, em setores diversos de um mesmo hospital, ou mesmo em instituições diferentes - propiciou-se integrar de forma informatizada diferentes profissionais envolvidos na ciência da vida e da saúde. Assim, criouse o termo bioinformática/ informática biomédica ${ }^{25}$.

O uso de computador em dietética teve seus primeiros registros na década de 1960. A Universidade Tulane em 1962 foi pioneira na pesquisa investigando o uso da tecnologia computadorizada em serviço de administração de alimentos ${ }^{30}$. Entre 1958 e 1983, foram relatadas mais de 400 referências da utilização de computador em serviços de alimentação ${ }^{10}$. Muitos dos artigos originais em nutrição e computação tratam da redução de custos e desperdício dos alimentos e redundância de dados nos departamentos de nutrição ${ }^{11}$. $\mathrm{Na}$ maioria dos casos, estes sistemas foram criações personalizadas ou planilhas específicas para a facilidade prática ${ }^{16,24}$. Existe limitada documentação sobre eles. Com o advento do sistema operacional Windows (Microsoft Corp, Redmond, WA) em 1990, deu-se a oportunidade para desenvolvimento de software de nutrição mais específico que permitiu troca de dados. Empresas de software hoje continuam a atualizar programas e aplicativos que têm sido utilizados com sucesso em uma variedade de ambientes de nutrição ${ }^{15}$.

$\mathrm{Na}$ terapia nutricional a integração com a informática também vem se desenvolvendo. A busca pela redução nos erros de cálculos em soluções parenterais e enterais, bem como a facilidade e redução no tempo de prescrição estimulou o desenvolvimento de vários softwares capazes de melhorar a eficiência das equipes de terapia nutricional $\left.\right|^{4,14,23}$.

O uso de protocolos é prática comum em muitas instituições. Eles podem ser construídos em papel ou utilizando recursos informatizados. Na saúde ele é utilizado para padronizar e uniformizar os procedimentos e informações do atendimento ao doente. Ele deve ser capaz de captar e armazenar dados, possibilitando controle, análise, acompanhamento, além de contribuir para tomada de decisão mais segura e eficaz ${ }^{28,13,6}$. Protocolos eletrônicos auxiliam para redução de erros em coletas de dados, diminuição da quantidade de papel e os seus custos relativos, e ter uso multicêntrico².

O Sistema Integrado de Protocolos Eletrônicos $(\operatorname{SINPE} C)^{21}$ foi desenvolvido visando solucionar algumas deficiências tipicamente encontradas em pesquisas, como falta de padronização dos termos; dados de uma pesquisa não utilizados em outra; dificuldades à realização de pesquisa multicêntrica e multidisciplinares ${ }^{18}$. Ele proporciona informações automáticas, instantâneas e ágeis. O armazenamento estruturado de dados clínicos facilita sua utilização. Proporciona mais confiabilidade à banco de dados clínicos prospectivo, com possibilidade de resgate e cruzamento das informações. Visa, também, a produção de estudos científicos de alta qualidade com credibilidade e menor tempo ${ }^{20}$.
A assistência domiciliar é prática que remonta à era a.C. Porém, somente em meados do século XIX começaram a surgir as primeiras sistematizações das atividades relacionadas ao serviço de enfermagem. Em 1790 foi instituído por assistentes sociais e outros profissionais o Home Care em Hospital de Boston. Este modelo de assistência permanece até os tempos atuais, com visitas realizadas por equipes multiprofissionais ${ }^{3}$.

No Brasil o desenvolvimento da assistência domiciliar se deu no começo do século XX, em 1919, com a criação do Serviço de Enfermeiras Visitadoras, no Rio de Janeiro ${ }^{3}$. A Terapia Nutricional Domiciliar (TND), como modalidade de atendimento em saúde, começou a se destacar na década de 80. Desde então, é cada vez maior a tendência de se prosseguir em nível domiciliar, com os cuidados hospitalares envolvendo, além da TND, a terapia nutricional enteral domiciliar (TNED) e terapia nutricional parenteral.

É importante dispor dos registros dos pacientes em TND sendo que estas informações permitem coordenar os recursos sanitários e melhorar a assistência ${ }^{22}$. A prática cotidiana, no entanto, tem sido crescente e os dados e informações pouco conhecidos. Justifica-se assim a necessidade de criação de recursos mais atuais para manipulação e processamento de informações.

O objetivo deste estudo é elaborar um protocolo eletrônico para o atendimento de pacientes em terapia nutricional enteral domiciliar.

\section{MÉTODO}

A construção do protocolo eletrônico em TNED foi dividida em três grandes etapas.

Na primeira etapa realizou-se revisão da literatura sobre terapia nutricional enteral domiciliar. Após a revisão, foram levantados 1793 subitens, agrupados em nove setores principais (Figura 1). Deste modo, obteve-se a criação da base teórica de dados da terapia nutricional enteral domiciliar.

\begin{tabular}{|c|c|}
\hline Itens do protocolo & Quantidade de filhos do protocolo \\
\hline Identificação & 236 \\
\hline Avaliação nutricional & 1340 \\
\hline Prescrição dietética & 34 \\
\hline Indicações de nutrição enteral & 60 \\
\hline Vias de acesso para nutrição enteral & 9 \\
\hline Composição da nutrição enteral & 70 \\
\hline Sistema de administração & 10 \\
\hline Complicações & 18 \\
\hline Reinternamentos & 6 \\
\hline
\end{tabular}

FIGURA 1 - Itens principais do protocolo mestre

A segunda etapa foi a transferência dessa base teórica para a forma de protocolo eletrônico geral que absorvia todos os dados da base. Este protocolo, chamado foi chamado de Protocolo Mestre Informatizado de Terapia Nutricional Enteral Domiciliar. Ele foi incluído no já existente Sistema Integrado de 
Protocolos Eletrônicos (SINPEC). Foi desenvolvido na linguagem C\# (C-Sharp) utilizando plataforma NET Microsoft $\AA$ e o sistema de banco de dados Access ${ }^{\circledR}$ que possibilita a distribuição dos dados por meios magnéticos (CD-ROM ou pen-drive).

$\mathrm{O}$ protocolo mestre baseia-se em um conjunto de dados dispostos de forma hierarquizada, os quais são subdivididos em itens e subitens, e distribuídos em diferentes gerações - denominados "irmão" e "filho" - que definem a formatação do conteúdo da base teórica. O SINPE@ permite a visualização dos itens em estrutura de árvore. O sinal positivo (+) à esquerda do item, significa que ele possui filhos (subitens) e pode ser aberto (Figura 2).

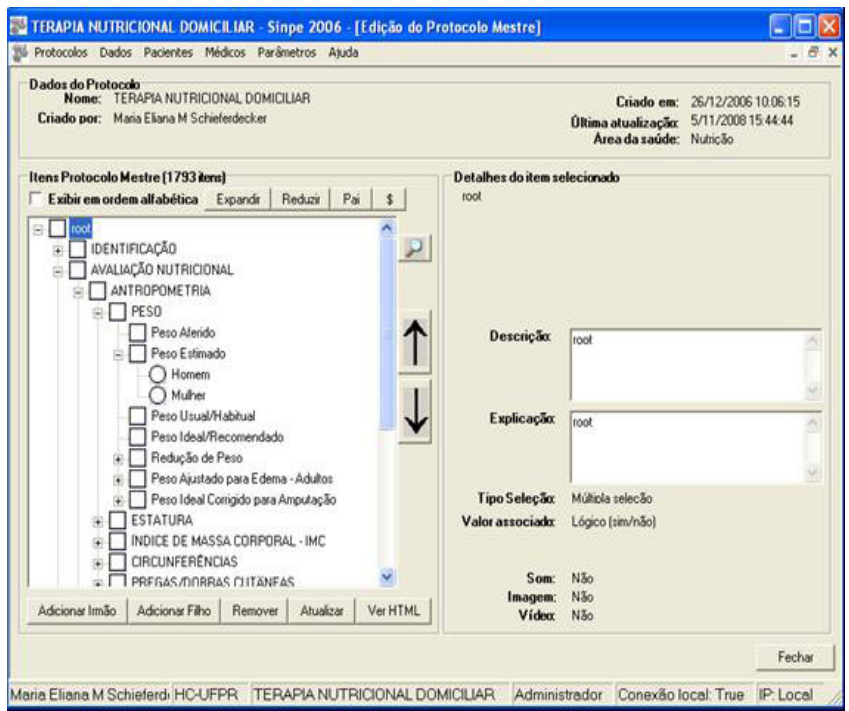

FIGURA 2 - Hierarquização do protocolo de atendimento nutricional domiciliar

A terceira e última etapa foi a criação de um protocolo específico a partir do protocolo mestre. Ele permite a seleção dos itens que têm relevância para a pesquisa que se pretende efetuar. Para criar os itens do protocolo específico usou-se o comando da seta direcionada para a direita. Após selecionar os itens e subitens contidos no protocolo mestre que interessam, a seta no sentido para à direita, uma vez clicada, leva a seleção de itens para o quadro da direita que é o que compõe o protocolo específico (Figura 3).

\section{RESULTADOS}

Depois de instalado por meio de um CD-ROM, o programa aparece na tela inicial com a presença do ícone SINPE $\odot$. Ao se clicar nele aparecerá na tela a janela que define o tipo de conexão que o usuário deseja. Se for local, a conexão será com a base em dados local utilizando o Microsoft Access ${ }^{\circledR}$; se for remota, haverá a necessidade da internet para a conexão em um servidor de banco de dados Microsoft SQL Server ${ }^{\circledR}$.
Após a escolha da conexão, deve ser selecionada a opção "Avançar". Ao avançar na seleção da conexão o programa solicita a identificação através de um usuário, senha e instituição pré-definidos no SINPEC. O sistema reconhece o usuário com a identificação de segurança definida pelo tipo de autorização e disponibiliza a tela para seleção do protocolo mestre que o usuário poderá acessar (Figura 4).

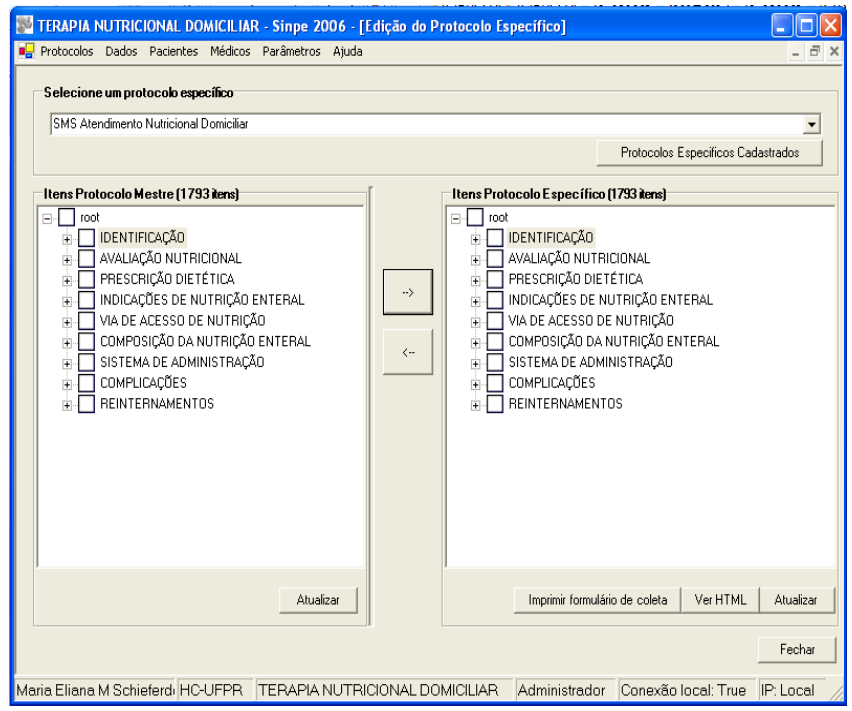

FIGURA 3 - Protocolo específico de atendimento nutricional domiciliar da Secretaria Municipal de Saúde de Curitiba

Seleção do Protocolo Mestre

\section{Olá Maria Eliana M Schieferdecker}

Você é um SUPER USUARIO, selecione o protocolo mestre que deseja ou crie um novo.

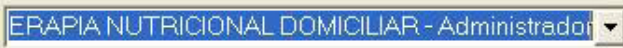

Protocolos Cadastrados

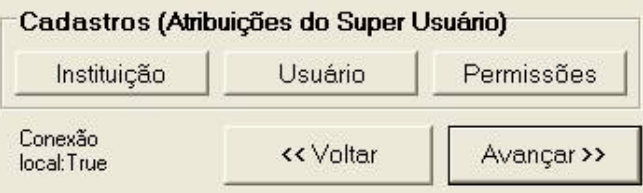

FIGURA 4 - Seleção do protocolo mestre

$\mathrm{Na}$ barra de ferramentas, na parte superior da tela principal consta o menu com as aplicações que podem ser feitas: protocolos, dados, pacientes, médicos, parâmetros e ajuda. Ao selecionar a opção "Protocolos", o usuário administrador terá acesso ao protocolo mestre ou ao protocolo específico.

Ao ser selecionado o protocolo específico serão apresentadas as categorias que o compõe. Clicando no 
sinal de "+" ao lado da categoria escolhida haverá a expansão deste item, abrindo os subitens como mostra a Figura 5.

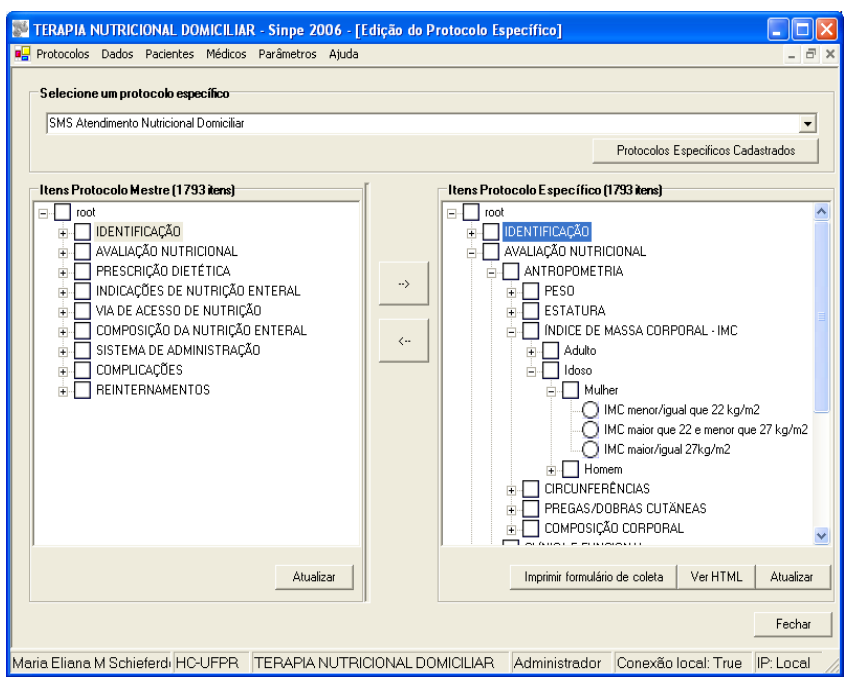

FIGURA 5 - Edição do protocolo específico

Os itens que compõe o protocolo específico estão contidos no Protocolo Mestre e as informações referentes a este protocolos são visualizados acima das caixas de diálogo, na esquerda o nome e a quantidade de itens do Protocolo Mestre e a direita a indicação que é um protocolo específico e seu respectivo numero de itens.

Os demais itens do menu não foram abordados nesta etapa do trabalho.

\section{DISCUSSÃO}

A padronização na terapia nutricional é importante no tratamento e definição da atuação dos membros da equipe. Isto tornou-se possível no Brasil após a publicação da Portaria SVS/MS n 337 de 1999 e posteriormente substituída pela Resolução 63 de 2000 do Ministério da Saúde que normatiza a terapia nutricional enteral. Estas normas determinam a necessidade de protocolos em todos os serviços de terapia nutricional. Além da padronização que define as ações dos profissionais é possível estabelecer os indicadores que controlam a qualidade.

Na revisão de Adam $^{1}$, quando analisa os benefícios que um protocolo pode trazer para o fornecimento de suporte nutricional, reforça-se a possibilidade de melhorar a qualidade, garantindo boas práticas, permitindo que o doente seja tratado no seu problema específico com foco na resposta, e fornecer base de dados para a auditoria, pesquisas e elaboração de diretrizes para condutas em saúde.

Certamente a transição de registro em papel para eletrônico dos protocolos traz grandes vantagens na redução de espaço físico necessário para arquivamento dos documentos ${ }^{13,19}$; no número de pessoas necessárias para o arquivamento; nos custos a longo prazo ${ }^{25}$; na facilidade no armazenamento e posterior captura dos dados clínicos; na maior confiabilidade dos dados coletados ${ }^{13,26 ;}$; no armazenamento de dados; na produção de estudos com grandes séries com informações fidedignas. Substancia, assim, a produção de diretrizes, 29 .

Apesar do desenvolvimento da informática na nutrição ter-se iniciado na década de $1960^{\circ}$, os benefícios que ela proporciona ainda são poucos. Estudo da American Dietetic Association's (ADA's) em 2007, com inquérito envolvendo 55.063 e-mails e taxa de resposta de $20,4 \%$, mostrou que: os nutricionistas utilizam tecnologia básica (e-mail e internet) e que o conceito de nutrição e informática é novo e pouco compreendido na área. Contudo, o número expressivo de respostas (11 000) indicou grande interesse da informática na nutrição ${ }^{4,19}$.

Um dos pontos cruciais para efetivação dos registros eletrônicos - a segurança dos dados armazenados - já foi superado. Atualmente existe no Brasil normatização detalhada a respeito do assunto, com objetivo de salvaguardar a confidencialidade dos dados clínicos ${ }^{7}$.

Com relação a essa questão, o SINPE $@$ tem especial preocupação, apresentando várias ferramentas que possibilitam confidencialidade e proteção dos dados inseridos. Isso fica evidenciado pela diferenciação entre usuários, tipos de permissão outorgada a cada um, bem como a impossibilidade de alteração no protocolo, uma vez que tenha sido efetuada coleta.

O controle e padronização dos dados nos protocolos permitem estudos prospectivos e longitudinais ${ }^{12}$. O número de paciente em TNED está aumentando em todo o mundo. Tradicionalmente os profissionais individualmente atendem estes pacientes e detalhes de seu manejo nutricional são mantidos no prontuário dos registros.

$\mathrm{Na}$ elaboração do protocolo eletrônico foram criados 1793 subitens agrupados em nove itens principais. Procurou-se contemplar todos indicadores para avaliação nutricional, prescrição e acompanhamento da TNED.

Encontrou-se na literatura científica um único estudo publicado em 2008 como abstract do " $23^{\text {rd }}$ Annual Congress of the Czech Society for Clinical Nutrition and Intensive Metabolic Care" com interesse de coletar informações sobre pacientes que receberam TNED e desenvolveram uma base de dados. Após verificar as diretrizes correntes na administração e acompanhamento dos pacientes e as opiniões dos nutricionistas dos hospitais envolvidos, foi criado banco de dados utilizando o Microsoft Access $2000^{\mathrm{TM}}$. O banco desenvolvido continha 10 diversas janelas de entrada. Foram gerados os relatórios da situação sociodemográficas, estado nutricional, tipo de terapia nutricional, diagnóstico principal, principal razão para da terapia nutricional e fórmula utilizada pelos 
pacientes. Em 2008 na Austrália foi criada a primeira base de dados para a obtenção das informações sobre pacientes recebendo TNED em um serviço de saúde ${ }^{27}$.

A informatização tem demonstrado que ocorre grande diminuição da carga de trabalho ${ }^{17,8}$, proporciona apoio na decisão ${ }^{5}$ e pode melhorar a prescrição e a segurança nos cuidados com o paciente.

\section{CONCLUSÃO}

Foi possível criar um protocolo eletrônico para o atendimento de pacientes em terapia nutricional enteral domiciliar com dados seguros que contribuem para a pesquisa nesta área. Quando analisado pelo SINPEC, este aplicativo proporciona coleta, análise e a mineração de dados.

\section{REFERÊNCIAS}

1. Adam S. Standardization of nutritional support: are protocols useful? Intensive and Critical Care Nursing 2000;16(283-289).

2. Afrin LB, Kuppuswamy V, Slater B, Stuart RK. Electronic Clinical Trial Protocol Distribution via the World-Wide Web: A Prototype for Reducing Costs and Errors, Improving Accrual, and Saving Trees. Am Med Inform Assoc1997;4:25-35.

3. .Albuquerque SMRL. Qualidade de vida do idoso: A assistência domiciliar faz a diferença? Cedecis; São Paulo2003. p. 159.

4. Ayres EJ, Hoggle LB. ADA Nutrition Informatics Member Survey: Results and Future Steps. J Am Diet Assoc.2008;108(11):1822-4.

5. Bates DW, Teich JM, Lee J, Seger D, Kuperman GJ, Ma'Luf N. The impact of computerized physician order entry on medication error prevention. J Am Med Inform Assoc1999;6:313-21.

6. Berger $C$, et al.Coleta de dados eletrônicos para análise das manobras cirúrgicas em pacientes submetidos a rinoplastia. Int. Arch. Otorhinolaryngol. 2012;16(4):497-501.

7. Borsato EP. Modelo multicêntrico para elaboração, coleta e pesquisa de dados em protocolos eletrônicos. Curitiba: UFPR; 2005.

8. Bosman RJ, Oudemans VSHM, Zandstra DF. The use ofintensive care information systems alters outcome prediction. Intensive Care Med1998;24:953-8.

9. Dorr $D$, et al. Informatics Systems to promote improved care for chronic illness: a literature review. J Am Med Inform Assoc. 2007; 14:156-63.

10. Fowler KD. Evaluating foodservice software: A suggested approach. J Am Diet Assoc.1986;86:1224-7.

11. Fromm B, Moore AN, Hoover LW. Computer-generated fiscal reports for food cost accounting. J Am Diet Assoc.1980;77:170-8.
12. Grimson J. Delivering the electronic healthcare record for the 21st century. International Journal of Medical Informatics.2001; 64:111-27.

13. Haux Rea. Health care in the information society. A prognosis for the year 2013. Int J Med Inf.2002;66:3-21.

14. Hoggle LB, Michael MA, Houston SM, Ayres EJ. Electronic Health Record: Where Does Nutrition Fit In? J Am Diet Assoc.2006;106(10):1688-95.b.

15. Hoggle LB, Michael MA, Houston SM, Ayres EJ. Nutrition Informatics. J Am Diet Assoc.2006;106(1):134-9.

16. Hoover LW, Meylor DJ. Clinical dietitians use computer-generated worksheet. J Am Diet Assoc.1976;69:404-5.b.

17. KarI A. CIS in the ICU-fears and experiences. Int J Intensive Care.1998;5:15-7.

18. Malafaia O, Dietz UA, Ribas Filho JM, Czeczko NG, Borsato EP, Pinto JS. Elektronische Datenerfassung im Bereich der klinischen Forschung, Lehre und ärztlichen Verwaltung in dem Krankenhaus der Zukunft.2006.

19. Mikkelsen G, Aasly J. Concordance of information in parallel eletronic and paper based patient records. Int $\mathrm{J}$ Med Inf.2001;63:123-31.

20. Oliveira MM, Moraes RS, Matias JEF, Pinto JSP, Trippia MA, Kurestki C, Coelho JCU. Análise Informatizada dos Resultados Obtidos pela Aplicação de Protocolo Eletrônico na Microcirurgia Endoscópica Transanal (Tem). ABCD Arq Bras Cir Dig. 2010;23(1):29-34

21. Pinto JS. Interfase de visibilização de informações para o sistema integrado de protocolos eletrônicos. Curitiba: UFPR; 2005.

22. Planas Mea. Registro Nacional de la Nutrición Enteral Domiciliaria (NED) del año 2003. Nutr Hosp.2006;21(1):71-4.

23. Puangco MA, Nguyen HL, Sheridan MJ. Computerized PN ordering optimizes timely nutrition therapy in a neonatal intensive care unit. J Am Diet Assoc.1997;97(3):258-61.

24. Seay R. Spreadsheet for calculating nutritional intake in pediatric patients. Am J Health Syst Pharm.1996;53:663-7.

25. Shortliffe EH, Cimino JJ. Biomedical informatics. Computer applications in health care and biomedicine. 3. ed. New York: Springer Science+Business Media; 2006.

26. Uslu AM, Stausberg J. Value of the electronic patient record: an analysis of the literature. J Biomed Inform.2008;41(4):675-82.

27. Vozzo R, LO J, Lier G, Byrne M, Mcqueen J, Talbot P. Development of a Home Enteral Nutrition (HEN) Patient Database for Sydney West Area Health Service. Nutrition. 2008;24(5):512.

28. Walach VR. Aplicabilidade do Sistema Integrado de Protocolos Eletrônicos - SINPE - como ferramenta gerencial na análise de custos de procedimentos de revascularização do miocárdio no Hospital de Clínicas da Universidade Federal do Paraná. Curitiba: UFPR; 2008.

29. Young A Sea. Information technology to support improved care for chronic illness. J Gen Intern Med. 2007;22(3):425-30.

30. Youngwirth J. The evolution of computers in dietetics: A review. J Am Diet Assoc.1983;82:62-7. 